\title{
ANÁLISE DE DESEMPENHO DO FLICKERMETER NA PRESENÇA DE COMPONENTES INTER-HARMÔNICAS
}

\author{
José Rubens Macedo Jr.* \\ jrubens@ieee.org
}

\author{
Domingos S. L. Simonetti ${ }^{\dagger}$ \\ d.simonetti@ele.ufes.br
}

*Faculdade de Engenharia Elétrica, UFU, Av. João Naves de Ávila, 2121,

Campus Santa Mônica, Uberlândia - MG

†Departamento de Engenharia Elétrica, UFES,

Av. Fernando Ferrari, s/n,

Campus Universitário, Vitória - ES

\begin{abstract}
Performance analysis of flickermeter in the presence of interharmonic components

Until the beginning of the first decade of this century it was believed that the appearance of the phenomenon of voltage fluctuation, and eventually flicker, was necessarily related to the abrupt changes, repetitive or random, in reactive power required by some loads like, for example, electric arc furnaces and hot strip mills. Concomitantly, the IEC flickermeter protocol was developed in this specific context. In this sense, the present paper shows some simulation results, as well as results obtained in the laboratory, showing problems in the quantification of voltage fluctuations by the IEC flickermeter, when interharmonic voltages are superimposed on the fundamental frequency.
\end{abstract}

KEYWORDS: Flickermeter, interharmonics.

\section{RESUMO}

Até o início da primeira década do século XXI acreditava-se que o aparecimento do fenômeno da flutuação de tensão, e eventualmente da cintilação luminosa (flicker), estava necessariamente relacionado com a variação abrupta, repetitiva ou

Artigo submetido em 02/04/2011 (Id.: 1315)

Revisado em 30/05/2011, 19/10/2011

Aceito sob recomendação do Editor Associado Prof. Francisco de Assis dos Santos Neves aleatória, da potência reativa requerida por algumas cargas elétricas específicas como, por exemplo, os fornos elétricos a arco e os laminadores de tiras. E foi exatamente nesse contexto que o protocolo da IEC para quantificação dos níveis de severidade de flicker foi desenvolvido. Nesse sentido, o presente trabalho mostrará alguns resultados de simulações computacionais, assim como resultados obtidos em laboratório, evidenciando problemas na quantificação das flutuações de tensão através do flickermeter IEC, quando da sobreposição de tensões com frequências inter-harmônicas ao sinal de tensão fundamental.

PALAVRAS-CHAVE: Flickermeter, inter-harmônicas.

\section{INTRODUÇÃO}

Originalmente, o fenômeno da flutuação de tensão e, particularmente da cintilação luminosa (flicker), estava diretamente relacionado com a operação de cargas cujas características de operação resultavam em solicitações bruscas, repetitivas ou aleatórias, das componentes de potência reativa. Com os avanços tecnológicos, no entanto, notadamente relacionados com uma maior difusão das cargas eletrônicas de dupla conversão como, por exemplo, os sistemas de controle de velocidade de motores elétricos e as lâmpadas fluorescentes compactas, novas formas de aparecimento do fenômeno da cintilação luminosa passaram a ser amplamente estudadas (Macedo Jr. and Simonetti, 2009)(Li et al., 2003). Nesse sentido, as componentes de tensão com frequências inter- 
harmônicas ganharam um papel de destaque, quebrando, inclusive, antigos paradigmas da engenharia elétrica.

Um aspecto muito importante relacionado com a questão das tensões com frequências inter-harmônicas diz respeito à baixa amplitude e a intermitência das mesmas. Contudo, para o efeito da cintilação luminosa, bastam apenas pequenas amplitudes de tensões com frequências inter-harmônicas, sobrepostas ao sinal de tensão fundamental, para que o fenômeno da cintilação seja perfeitamente visível em algumas situações (Macedo Jr. and Simonetti, 2009). Para esse efeito, inclusive, essas amplitudes podem ser tão pequenas a ponto de serem quantificadas em partes por mil ao invés dos tradicionais valores percentuais.

Fundamentalmente, o flickermeter IEC baseia-se na amplitude e frequência de modulação dos valores eficazes das tensões (IEC, 2003). Contudo, algumas lâmpadas eletrônicas modernas reproduzem o fenômeno da cintilação luminosa em função da modulação do valor de pico da onda de tensão, mesmo quando a amplitude de modulação do valor eficaz da tensão aplicada é desprezível. As inter-harmônicas de frequências superiores a $200 \mathrm{~Hz}$, em geral, promovem uma modulação significativa do valor pico da tensão, sem apresentar modulação apreciável no valor eficaz do mesmo sinal. Dessa forma, o fato do flickermeter IEC ser totalmente fundamentado no cálculo do valor eficaz da tensão resulta em deficiências intrínsecas do protocolo da IEC quando da monitoração de ambientes nos quais sejam verificadas componentes inter-harmônicas sobrepostas à tensão fundamental da rede. Finalmente, os filtros digitais implementados no bloco 3 do flickermeter IEC (IEC, 2003) também contribuem para algumas falhas intrínsecas do protocolo IEC, conforme será mostrado mais adiante.

\section{FUNDAMENTOS TEÓRICOS}

Para uma melhor compreensão das deficiências do flickermeter IEC, quando da presença de componentes interharmônicas, torna-se oportuno ilustrar as diferenças conceituais entre a frequência inter-harmônica sobreposta ao sinal fundamental e a frequência de modulação do respectivo sinal resultante. Nesse sentido, a sobreposição de uma componente inter-harmônica sobre a onda de tensão fundamental pode suscitar, eventualmente, o entendimento de que a frequência de modulação da onda de tensão resultante seja exatamente igual à frequência da componente interharmônica sobreposta. Porém, esse não é o entendimento correto sobre a questão.

Para ilustrar esse fato, a figura 1 mostra as frequências de modulação do valor de pico da onda resultante, obtida quando da sobreposição individual de duas componentes inter-harmônicas distintas (todas com amplitudes iguais a
$10 \%$ em relação à fundamental) com frequências de 80 e 90 $\mathrm{Hz}$, respectivamente.
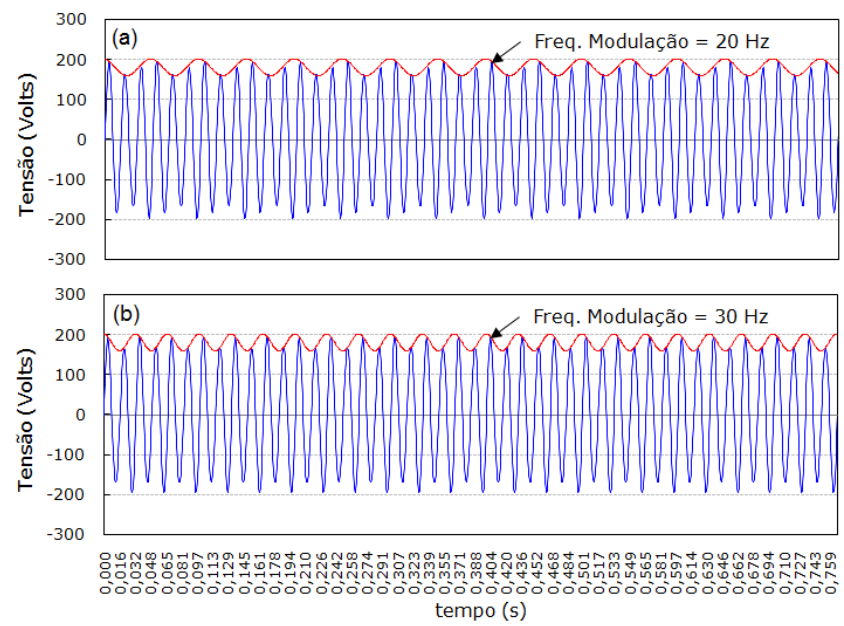

Figura 1: (a) Inter-harmônica de $80 \mathrm{~Hz}$ sobreposta ao sinal de tensão fundamental em $60 \mathrm{~Hz}$; (b) Inter-harmônica de 90 $\mathrm{Hz}$ sobreposta ao sinal de tensão fundamental em $60 \mathrm{~Hz}$.

Como pode ser observado na figura 1 , as frequências de modulação são totalmente diferentes das frequências das componentes inter-harmônicas sobrepostas ao sinal de tensão fundamental. Para o caso da figura 1(a), a frequência de modulação do valor de pico da onda de tensão é de $20 \mathrm{~Hz}$, por sua vez, conforme mostrado na figura 1(b), a sobreposição de uma componente inter-harmônica com frequência de $90 \mathrm{~Hz}$ gera uma frequência de modulação no valor de pico da onda de tensão igual a $30 \mathrm{~Hz}$.

Analiticamente, este resultado pode ser traduzido através da equação (1), mostrada a seguir (Li et al., 2003).

$$
f_{\text {modulacao }}=\left|f_{i h}-f_{h}\right|
$$

Onde:

$f_{\text {modulacao }}=$ frequência de modulação;

$f_{i h}=$ frequência inter-harmônica sobreposta ao sinal fundamental;

$f_{h}=$ frequência harmônica mais próxima de $f_{i h}$.

Em termos práticos, a equação (1) diz que a frequência de modulação é igual ao módulo da diferença entre a frequência inter-harmônica, sobreposta ao sinal fundamental, e a frequência harmônica imediatamente adjacente. A equação (1) pode ser graficamente interpretada conforme mostrado na figura 2. Na mesma figura estão indicadas as frequências 
inter-harmônicas para a máxima percepção visual de flicker $(8,8 \mathrm{~Hz})$.

As frequências de modulação somente serão coincidentes com as frequências inter-harmônicas sobrepostas ao sinal fundamental no caso particular das componentes subharmônicas com frequências iguais ou inferiores a $30 \mathrm{~Hz}$. Assim, por exemplo, a sobreposição de uma componente inter-harmônica com frequência de $10 \mathrm{~Hz}$ produzirá uma modulação da tensão resultante nesta mesma frequência.

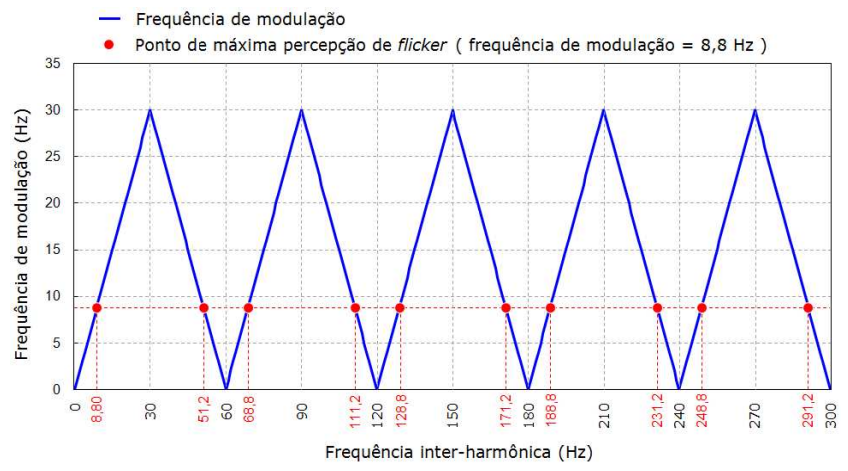

Figura 2: Frequências de modulação versus frequências inter-harmônicas.

Outra observação importante, também resultante da análise da figura 2, é que qualquer frequência inter-harmônica individual, sobreposta ao sinal de tensão fundamental, produzirá uma frequência de modulação dentro dos limites de percepção do olho humano $(0,5 \mathrm{~Hz}-30 \mathrm{~Hz})$. Evidentemente que a percepção do fenômeno da cintilação luminosa dependerá também da amplitude da componente inter-harmônica individual, assim como do tipo de lâmpada considerado.

Basicamente, as deficiências intrínsecas do flickermeter IEC na quantificação do fenômeno da cintilação luminosa, quando da presença de componentes inter-harmônicas nas redes de energia elétrica, estão relacionadas à modulação dos valores de pico e eficaz das ondas de tensão, assim como aos filtros digitais implementados em um dos blocos estruturais do protocolo IEC 61.000-4-15 (IEC, 2003). No presente trabalho, apesar da utilização do termo "deficiências intrínsecas" do flickermeter IEC, cabe ressaltar que não se trata de um erro conceitual do protocolo elaborado pela IEC, mas sim do fato de que o mesmo não é suficiente para quantificar corretamente o fenômeno da cintilação luminosa em determinadas condições, as quais serão mais bem analisadas nos tópicos a seguir.

\subsection{Modulação do valor eficaz e de pico da tensão resultante quando da presença de componentes inter- harmônicas}

Originalmente, o protocolo IEC 61.000-4-15 para quantificação dos indicadores de severidade de flicker foi desenvolvido considerando-se o fenômeno da cintilação luminosa em lâmpadas de filamento incandescentes. Dessa forma, temse que a variação do fluxo luminoso em uma lâmpada incandescente é dependente da temperatura e da inércia térmica de seu filamento. Adicionalmente, a temperatura do filamento está diretamente relacionada com a potência dissipada na lâmpada, o que equivale a dizer, finalmente, que o fluxo luminoso da lâmpada possui relação direta com a variação, ou modulação, da tensão eficaz aplicada aos terminais da lâmpada (IEEE, 2001); (Gallo et al., 2004). Assim sendo, o flickermeter IEC poderia ser deficiente para quantificar a sensação da cintilação luminosa em lâmpadas cuja luminosidade não fosse sensível às variações térmicas de um filamento. Ou seja, não fosse sensível às variações do valor eficaz da tensão aplicada. E é exatamente isso o que ocorre nas lâmpadas fluorescentes, notadamente nas modernas lâmpadas fluorescentes compactas. Estas lâmpadas possuem um fluxo luminoso muito mais sensível às variações do valor de pico da tensão aplicada. E no caso da presença de componentes inter-harmônicas de tensão é possível haver uma variação considerável do valor de pico da tensão resultante, mesmo quando seu valor eficaz não sofra variações apreciáveis. As figuras 3 e 4 ilustram os efeitos da sobreposição de componentes inter-harmônicas na tensão fundamental nos respectivos valores de pico e eficaz.

Como pode ser observado, para o caso particular da figura 3, tanto a variação da envoltória da tensão de pico quanto a variação do valor eficaz da tensão resultante são bastante nítidas. Em contrapartida, pela análise da figura 4, apesar da existência de variação da envoltória da tensão de pico, a variação do valor eficaz da tensão resultante é praticamente inexistente. Estas constatações serão muito importantes quando das análises relacionadas com as variações dos fluxos luminosos emitidos pelas lâmpadas incandescentes e fluorescentes compactas, conforme será mostrado mais adiante. Em complemento às figuras 3 e 4, a figura 5 mostra que a variação do valor eficaz da onda de tensão resultante, quando da sobreposição de uma componente inter-harmônica, é mais acentuada para as frequências inter-harmônicas imediatamente adjacentes à frequência fundamental e praticamente inexistente para as frequências maiores que $200 \mathrm{~Hz}$.

Dessa forma, quando da sobreposição de componentes interharmônicas de frequências elevadas ao sinal fundamental, o flickermeter da IEC apresentará valores muito pequenos (praticamente inexpressivos) para os indicadores Sensação Ins- 

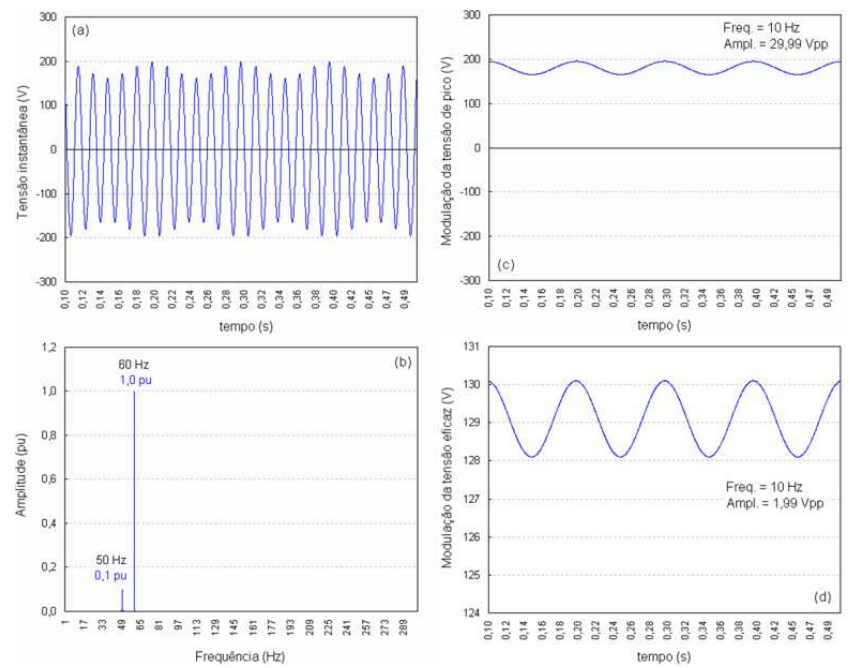

Figura 3: (a) Tensão resultante da sobreposição de uma componente inter-harmônica com frequência igual a $50 \mathrm{~Hz}$ e amplitude de $10 \%$ em relação à fundamental, (b) espectro de frequência da tensão resultante, (c) modulação da envoltória da tensão de pico e (d) modulação do valor eficaz.

tantânea de Flicker $(S f)$ e $P s t$, uma vez que não há variação do valor eficaz da onda de tensão resultante. Nos casos particulares das figuras 3 e 4, os valores da sensação instantânea de flicker registrados foram de 3225,0 pu e 0,24 pu, respectivamente. Já o indicador $P s t$ apresentou os valores de $\mathbf{4 1 , 3 3}$ $p u$ e $\mathbf{0 , 0 3 2} p u$, respectivamente.

Assim, resta saber se mesmo com os inexpressivos valores de $S f$ e Pst obtidos quando da sobreposição de componentes inter-harmônicas de frequências mais altas, como no caso da figura 4, algumas lâmpadas poderiam emitir alguma variação de luminosidade. Para responder a essa questão, foram elaborados testes de laboratório nos quais duas lâmpadas distintas, uma incandescente tradicional e outra fluorescente compacta equivalente em termos de luminosidade. A estrutura física dos testes realizados é mostrada na figura 6 .

Os resultados obtidos nos testes estão indicados na figura 7 , onde são mostradas as variações dos fluxos luminosos das lâmpadas quando da sobreposição de componentes interharmônicas de diferentes frequências sobre a componente de tensão fundamental.

Na figura 7 é possível observar que no caso da lâmpada incandescente a modulação do fluxo luminoso torna-se bastante inexpressiva quando da sobreposição das frequências mais altas. Em contrapartida, a modulação do fluxo luminoso registrada para a lâmpada fluorescente compacta continua apresentando amplitudes consideráveis, mesmo para as frequências mais altas. Para o caso específico da interharmônica de $172 \mathrm{~Hz}$, sobreposta ao sinal de tensão de ali-
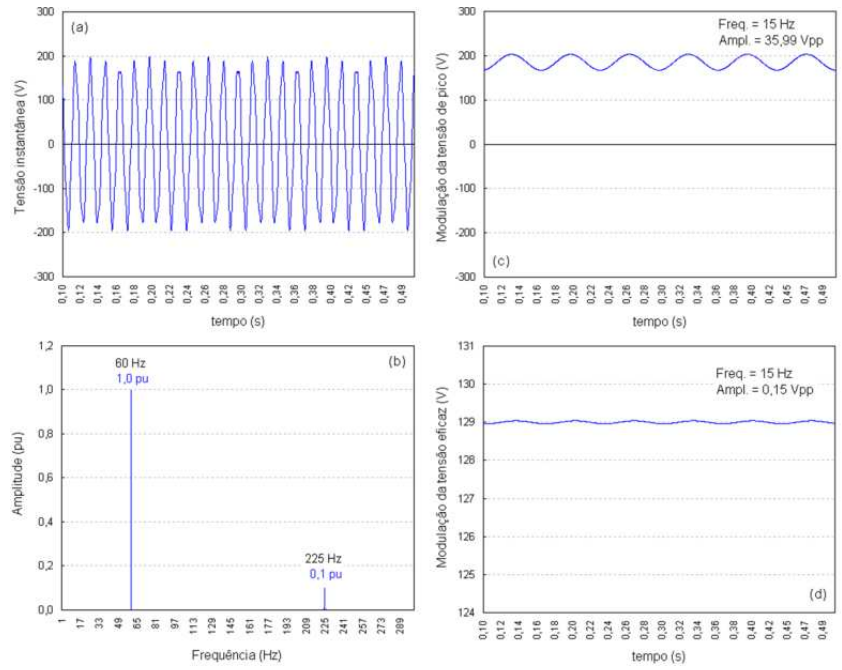

Figura 4: (a) Tensão resultante da sobreposição de uma componente inter-harmônica com frequência igual a $225 \mathrm{~Hz}$ e amplitude de $10 \%$ em relação à fundamental, (b) espectro de frequência da tensão resultante, (c) variação da envoltória da tensão de pico e (d) variação do valor eficaz.

mentação das lâmpadas, observou-se que o efeito da cintilação luminosa é bastante nítido para o caso da lâmpada fluorescente compacta e totalmente imperceptível para a lâmpada incandescente. Assim sendo, em termos práticos, os resultados apresentados na figura 7 evidenciam a maior sensibilidade da lâmpada incandescente à modulação do valor eficaz, ao passo que a lâmpada fluorescente compacta apresenta maior sensibilidade para modulação do valor de pico da tensão de suprimento. Outra constatação importante, ainda para o caso da sobreposição da frequência inter-harmônica de $172 \mathrm{~Hz}$ (e amplitude de 10\%), é que a sensação instantânea de flicker, medida nos terminais da lâmpada, foi de apenas 0,45 pu, apesar da nítida percepção visual do efeito da cintilação luminosa no caso particular da lâmpada fluorescente compacta. Cabe ressaltar ainda que segundo a metodologia da IEC (IEC, 2003), cerca de 50\% dos observadores humanos são capazes de perceber o efeito da cintilação luminosa apenas para registros de $S f$ iguais ou superiores a 1,0 pu.

Finalmente, considerando-se a grande disseminação das lâmpadas fluorescentes compactas ao redor do mundo, em substituição às lâmpadas incandescentes, pode-se dizer que os resultados obtidos evidenciam a necessidade de uma nova metodologia de quantificação das flutuações de tensão, em substituição ao atual protocolo definido pela IEC. 


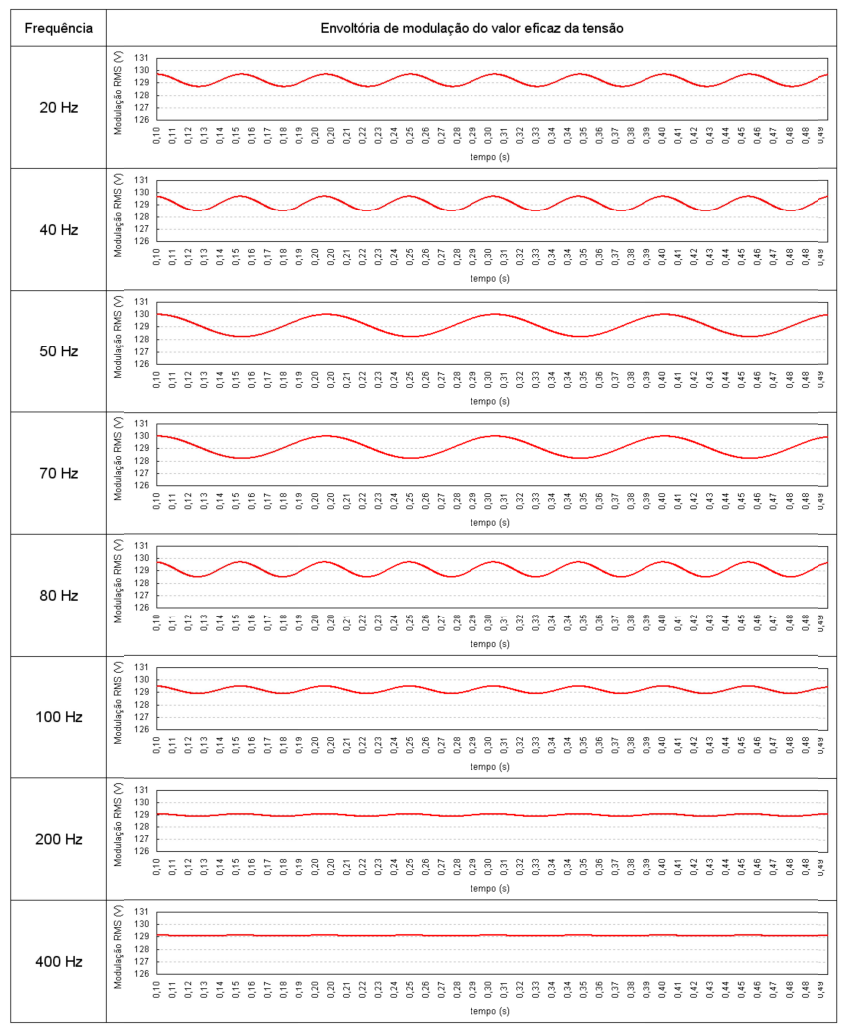

Figura 5: Modulação do valor eficaz da tensão resultante quando da sobreposição de frequências inter-harmônicas ao sinal de tensão fundamental.

\subsection{Análise da influência dos filtros digi- tais do flickermeter IEC em seu de- sempenho funcional.}

Além da questão da modulação verificada no cálculo dos valores eficazes da onda de tensão, sobreposta por componentes de tensão com frequências inter-harmônicas, os filtros digitais presentes no bloco 3 (ponderação em frequência) do flickermeter IEC, tipo passa-baixa e passa-alta, também exercem um papel importante nas deficiências do protocolo da IEC para quantificação dos indicadores de severidade de flicker na presença de inter-harmônicas. Para ilustrar esse fato, retomando-se à equação (1) e à figura 2 , espera-se que para uma mesma amplitude de componente inter-harmônica, os valores de Pst obtidos em função da superposição das frequências inter-harmônicas de 30, 90, 150, 210 e $270 \mathrm{~Hz}$ ao sinal de tensão fundamental, produzam exatamente o mesmo valor de severidade de flicker de curta duração (Pst). Afinal, nestes casos específicos, ter-se-ia uma mesma amplitude e uma mesma frequência de modulação $(30 \mathrm{~Hz})$ do valor eficaz da tensão resultante. No entanto, conforme mostrado na figura 8, os valores de Pst obtidos quando da sobreposição de frequências inter-harmônicas com amplitudes fixas de $10 \%$

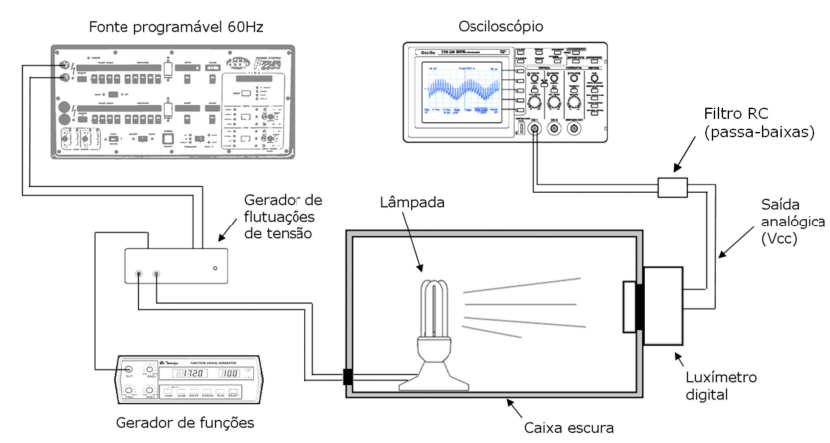

Figura 6: Estrutura de laboratório implementada para realização dos testes.

(em relação à fundamental), foram exatamente iguais apenas para o caso das inter-harmônicas com frequências de 30 e $90 \mathrm{~Hz}$. A partir da inter-harmônica com frequência de 150 $\mathrm{Hz}$ os valores de $P$ st registrados pelo flickermeter IEC foram totalmente inexpressivos.

Diante desta constatação, fica mais uma vez evidenciado que, a partir de uma determinada frequência inter-harmônica, sobreposta ao sinal de tensão fundamental, o flickermeter IEC não é capaz de quantificar de forma fisicamente correta os indicadores de severidade de flicker. Em parte, esse resultado foi explicado no tópico anterior como decorrência das baixas amplitudes de modulação do valor eficaz da tensão resultante, quando da sobreposição de componentes interharmônicas com frequências elevadas.

Adicionalmente à questão da modulação do valor eficaz, torna-se também importante relembrar a estrutura de filtros digitais utilizados no bloco 3 do flickermeter IEC (IEC, 2003). O flickermeter IEC é composto por três filtros, um do tipo passa-alta com frequência de corte igual a $0,05 \mathrm{~Hz}$, um segundo filtro do tipo passa-baixa (Butterworth de $6^{a}$ ordem) com frequência de corte igual a $42 \mathrm{~Hz}$ (curva para lâmpada de $120 \mathrm{~V} / 60 \mathrm{~Hz}$ ) e, finalmente, um terceiro filtro o qual constitui uma curva de ponderação em frequência. Este último, por tratar apenas da ponderação em frequência da perceptibilidade do olho humano quanto às flutuações de tensão, não apresenta influência no desempenho do flickermeter IEC e não será objeto de análise para os propósitos do presente tópico. A topologia dos dois primeiros filtros digitais utilizados no flickermeter IEC é apresentada na figura 9.

Conforme mostrado na figura 9, o sinal normalizado da tensão de entrada (bloco 1), após passar por uma demodulação quadrática (bloco 2), segue para os dois primeiros filtros do bloco 3 do flickermeter. Para um melhor entendimento desse processo, torna-se importante a realização de alguns desenvolvimentos algébricos para a correta identificação das com- 
ponentes de frequência envolvidas nas etapas indicadas na figura 9. Assim, o sinal injetado nos filtros é representado pelo sinal de tensão resultante em quadratura $\left(v^{2}\right)$, o qual pode ser algebricamente representado (Li et al., 2003)conforme equações a seguir.

$$
\begin{aligned}
& v=V_{1} \cdot \operatorname{sen}\left(2 \cdot \pi \cdot f_{1} \cdot t\right)+ \\
& \quad+V_{1} \cdot m \cdot \operatorname{sen}\left[2 \cdot \pi \cdot\left(f_{1}+\Delta f\right) \cdot t+\theta_{i h}\right]
\end{aligned}
$$

Onde:

$v=$ tensão resultante instantânea;
$V_{1}=$ valor de pico da tensão fundamental;

$f_{1}=$ frequência fundamental;

$m=$ Amplitude de modulação (\%);

$\Delta f=f_{i h}-f_{1}$;

$f_{i h}=$ frequência da componente inter-harmônica;

$\theta_{i h}=$ ângulo da componente inter-harmônica.

Fazendo-se (2) ao quadrado, resulta:

$$
\begin{aligned}
v^{2} & =V_{1}^{2} \cdot \operatorname{sen}^{2}\left(2 \cdot \pi \cdot f_{1} \cdot t\right)+V_{1}^{2} \cdot m^{2} \cdot \operatorname{sen}^{2}\left[2 \cdot \pi \cdot\left(f_{1}+\Delta f\right) \cdot t+\theta_{i h}\right]+ \\
& +V_{1}^{2} \cdot 2 \cdot m \cdot \operatorname{sen}\left(2 \cdot \pi \cdot f_{1} \cdot t\right) \cdot \operatorname{sen}\left[2 \cdot \pi \cdot\left(f_{1}+\Delta f\right) \cdot t+\theta_{i h}\right]= \\
& =\frac{1}{2} \cdot V_{1}^{2}-\frac{1}{2} \cdot V_{1}^{2} \cdot \cos \left(4 \cdot \pi \cdot f_{1} \cdot t\right)+V_{1}^{2} \cdot m \cdot \cos \left(2 \cdot \pi \cdot \Delta f \cdot t+\theta_{i h}\right)+ \\
& +V_{1}^{2} \cdot m \cdot \cos \left[2 \cdot \pi \cdot\left(2 \cdot f_{1}+\Delta f\right) \cdot t+\theta_{i h}\right]+ \\
& +\frac{1}{2} \cdot V_{1}^{2} \cdot m^{2}-\frac{1}{2} \cdot V_{1}^{2} \cdot m^{2} \cdot \cos \left[2 \cdot \pi \cdot\left(2 \cdot f_{1}+2 \cdot \Delta f\right) \cdot t+2 \cdot \theta_{i h}\right]
\end{aligned}
$$

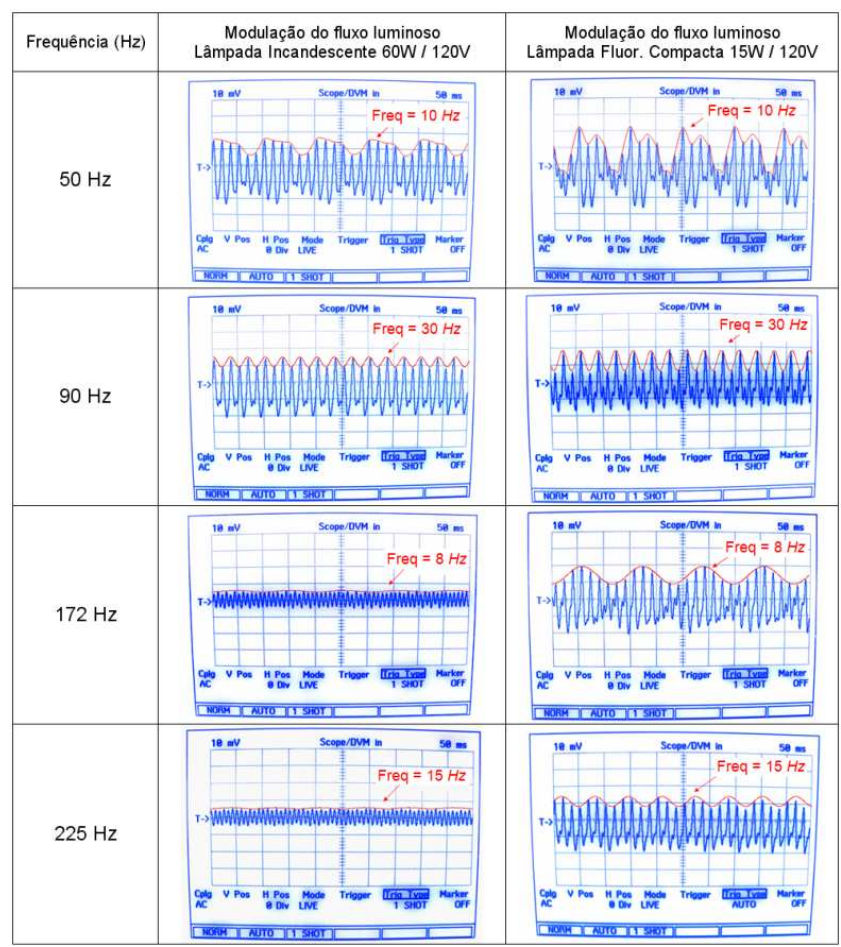

Figura 7: Modulação do fluxo luminoso em lâmpadas incandescentes e fluorescentes compactas.

Assim, ignorando-se o termo $\mathrm{m}^{2}$, resulta finalmente:

$$
\begin{aligned}
v^{2} & \approx \frac{1}{2} \cdot V_{1}^{2}-\frac{1}{2} \cdot V_{1}^{2} \cdot \cos \left(4 \cdot \pi \cdot f_{1} \cdot t\right)+ \\
& +V_{1}^{2} \cdot m \cdot \cos \left(2 \cdot \pi \cdot \Delta f \cdot t+\theta_{i h}\right)+ \\
& +V_{1}^{2} \cdot m \cdot \cos \left[2 \cdot \pi \cdot\left(2 \cdot f_{1}+\Delta f\right) \cdot t+\theta_{i h}\right]
\end{aligned}
$$

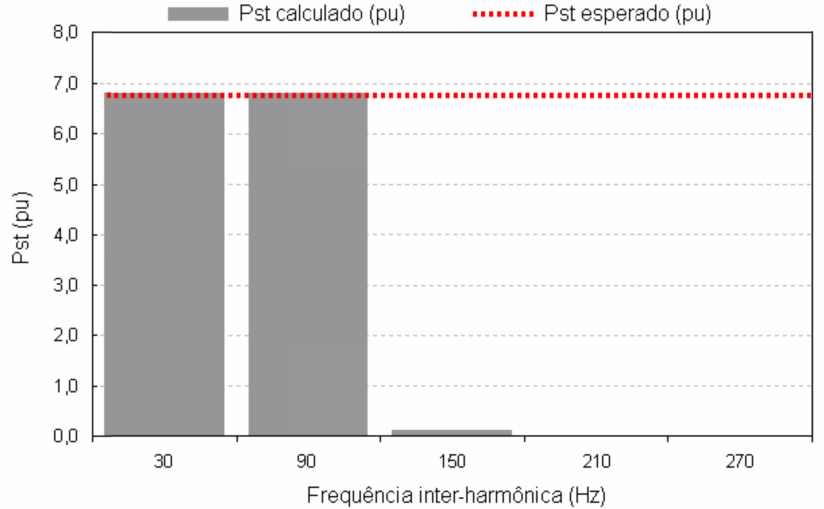

Figura 8: Cálculo do Pst para frequências inter-harmônicas de mesma magnitude e que produzam uma frequência de flicker igual a $30 \mathrm{~Hz}$.

Com base na aproximação indicada na equação (4), ficam evidentes quatro frequências específicas, as quais compõem o sinal de entrada para o bloco 3 (filtros digitais) do flickermeter IEC. São elas:

i. O nível $C C$ representado por $\frac{1}{2} \cdot V_{1}^{2}$;

ii. A componente representada por 2. $f_{1}$;

iii. A componente representada por $\Delta f$;

iv. E, finalmente, a componente representada por $2 . f_{1+} \Delta f$.

(4) Para uma melhor compreensão das componentes de frequência envolvidas com o processo, considera-se a seguir um 


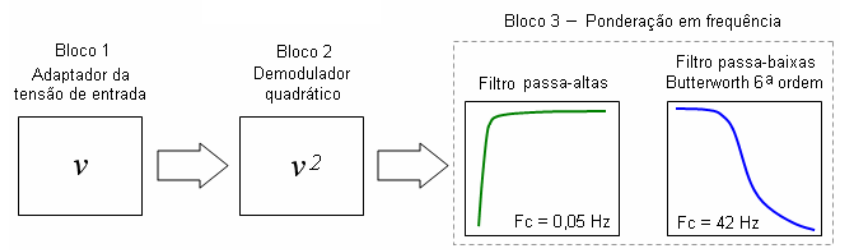

Figura 9: Topologia dos filtros digitais do flickermeter da IEC.

exemplo numérico no qual um sinal de tensão fundamental é sobreposto por uma componente de tensão inter-harmônica com frequência de $90 \mathrm{~Hz}$ e amplitude igual a 10\% da tensão fundamental.

$$
\begin{aligned}
v & =127 \sqrt{2} \cdot \operatorname{sen}(2 \pi \cdot 60 \cdot t)+ \\
& +127 \sqrt{2} \cdot 0,1 \cdot \operatorname{sen}[2 \pi \cdot(60+30) \cdot t+0]
\end{aligned}
$$

Comparando-se a equação (5) com a equação (2) ficam identificadas as seguintes variáveis: $V_{1}=127 \sqrt{2}$ Volts; $f_{1}=$ $60 \mathrm{~Hz} ; m=0,1 ; \theta_{i h}=0$ e $\Delta f=30 \mathrm{~Hz}$. Finalmente, fica também evidente que $f_{i h}=f_{1}+\Delta f=60+30=90 \mathrm{~Hz}$.

Aplicando-se a Transformada Discreta de Fourier ao sinal resultante da quadratura de (5), resulta o espectro mostrado na figura 10.

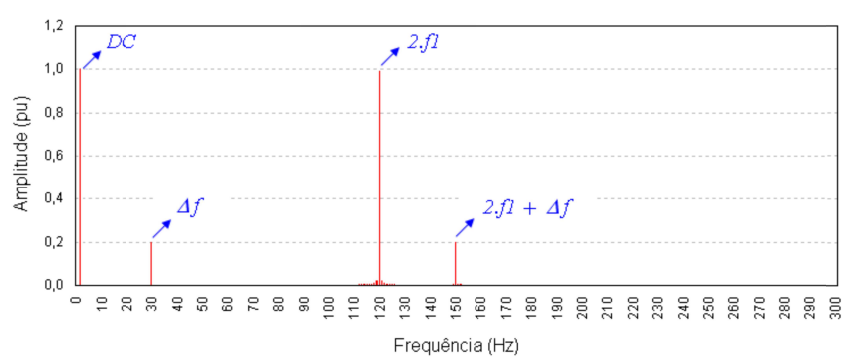

Figura 10: Espectro de frequência do sinal em quadratura da tensão resultante.

Conforme mostrado na figura 10, todas as frequências componentes do sinal em quadratura $v^{2}$ são perfeitamente identificadas no espectro de frequências associado. Assim, como contribuição adicional ao equacionamento algébrico sugerido em (Li et al., 2003), procede-se à sobreposição das envoltórias dos filtros tipo passa-alta e passa-baixa ao espectro mostrado na figura 10 . Nesse sentido, preliminarmente, a figura 11 apresenta o detalhamento das curvas de resposta em frequência dos referidos filtros.

Mesclando-se assim as curvas das figuras 10 e 11, conforme mostrado na figura 12 , tem-se uma visão clara do efeito dos dois primeiros filtros digitais do bloco 3 no desempenho do

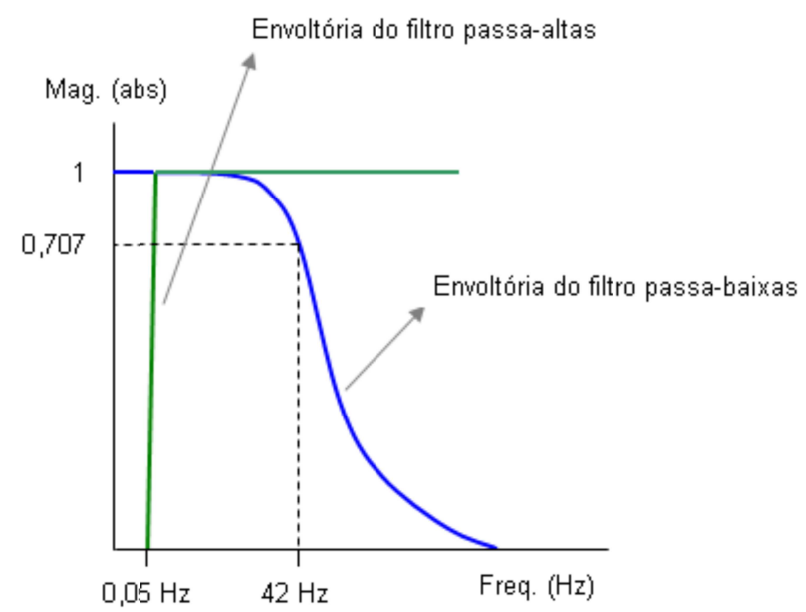

Figura 11: Resposta em frequência dos filtros tipo passabaixa e passa-alta do bloco 3 do flickermeter da IEC.

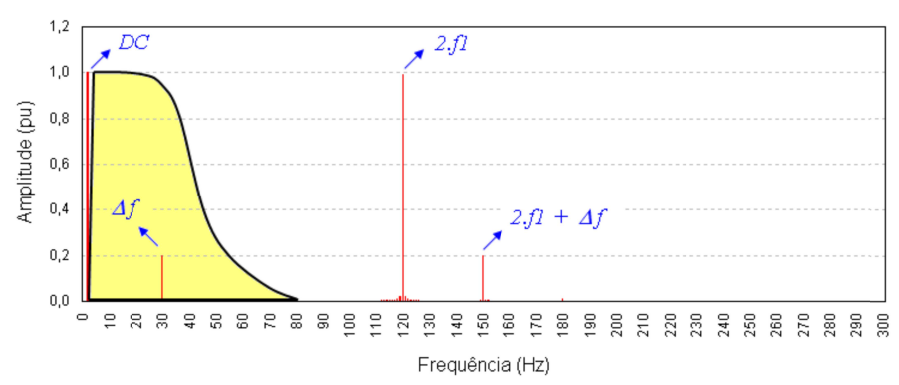

Figura 12: Espectro de frequência do sinal em quadratura da tensão resultante e envoltória dos filtros.

flickermeter IEC. De acordo com a figura 12, portanto, fica evidente que o sinal $C C$, a componente de frequência $2 . f_{1}$, assim como a componente de frequência $2 . f_{1}+\Delta f$ são definitivamente filtradas, não passando, portanto, para o próximo bloco do flickermeter IEC (bloco 4).

Assim, a única componente de frequência passível de avaliação é $\Delta f$. Portanto, a componente de frequência $\Delta f$ define, indiretamente, a máxima frequência da componente interharmônica que, sobreposta ao sinal de tensão fundamental, produzirá uma avaliação correta do indicador de severidade de flicker (Pst). Matematicamente, tem-se que $\Delta f$ será no máximo igual a $42 \mathrm{~Hz}$, frequência de corte do filtro passabaixa, o que equivale dizer que a máxima frequência interharmônica associada será $f_{i h} \leq 60+42=102 \mathrm{~Hz}$.

Finalmente, conclui-se que o flickermeter IEC não é capaz de detectar flicker causado pela sobreposição de componentes inter-harmônicas ao sinal de tensão fundamental, com frequência superior a $102 \mathrm{~Hz}$. Por esse motivo, os resulta- 

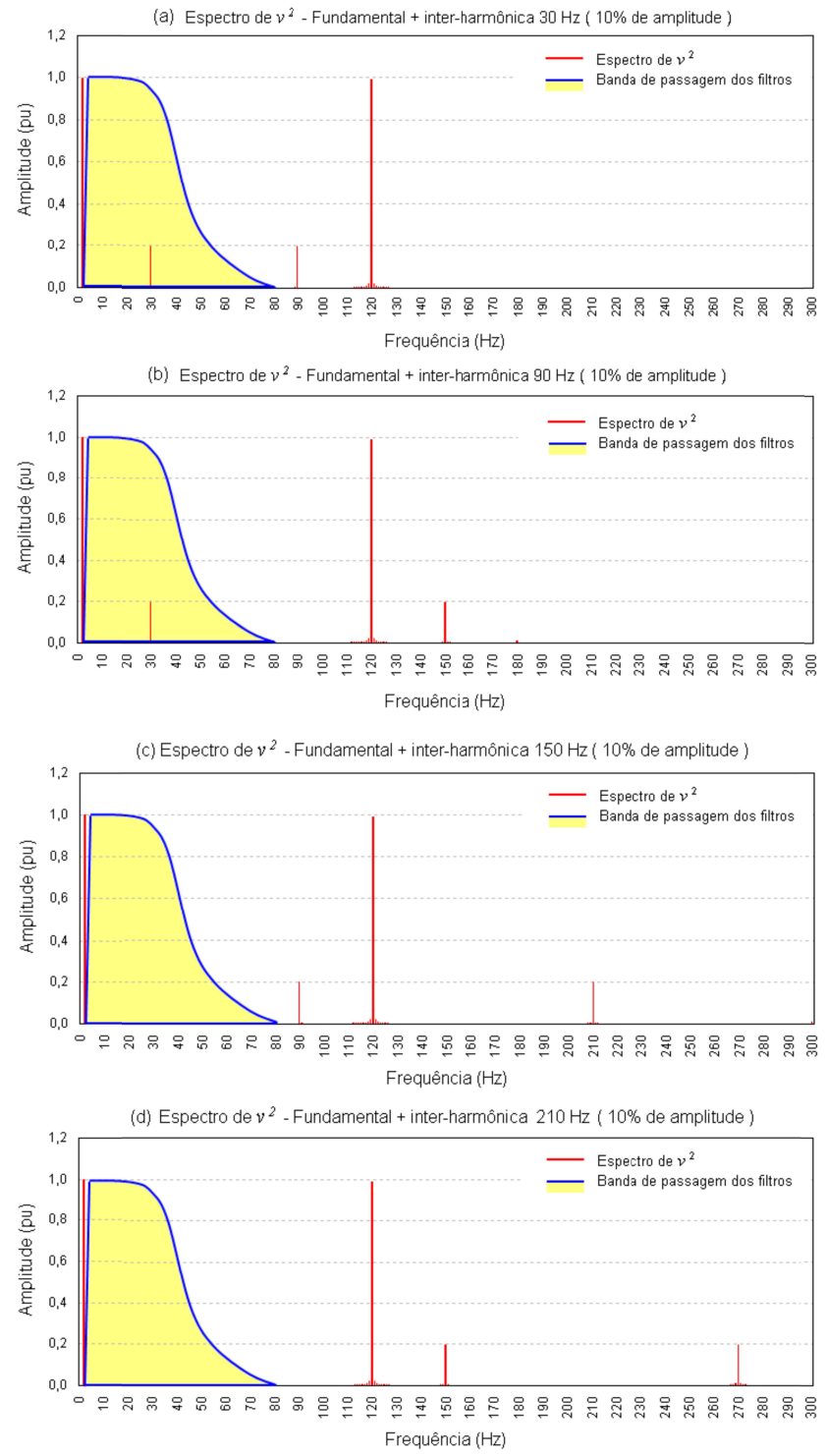

(e) Espectro de $v^{2}$ - Fundamental + inter-harmônica $270 \mathrm{~Hz}$ ( $10 \%$ de amplitude )

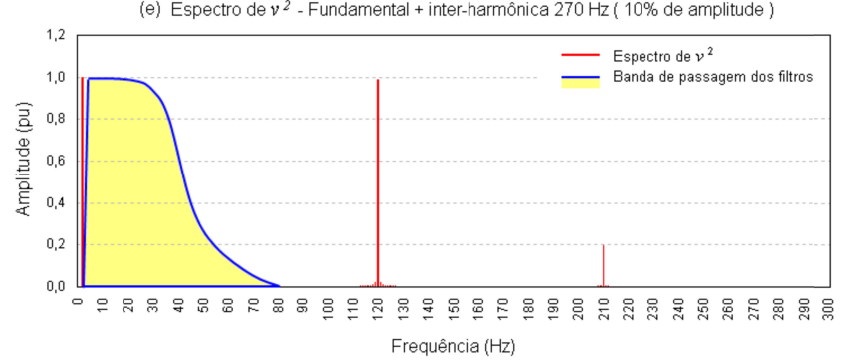

Figura 13: Espectro de frequência do sinal em quadratura da tensão resultante e envoltória dos filtros.

dos mostrados na figura 8 são tão discrepantes, quando na verdade deveriam ser exatamente iguais.
A figura 13 mostra a sobreposição dos espectros de frequência com as envoltórias dos filtros digitais para cada um dos casos analisados na figura 8. Como poderá ser observado, apenas para o caso das frequências inter-harmônicas de 30 e $90 \mathrm{~Hz}$ a componente $\Delta f$ não é eliminada pelos filtros, resultando, portanto, em uma correta avaliação do indicador $P$ st. Para os demais casos $\left(f_{i h}=150,210\right.$ e $\left.270 \mathrm{~Hz}\right)$, a componente $\Delta f$ é totalmente eliminada pelos filtros, mais especificamente pelo filtro passa-baixa, não sendo possível a correta apuração do indicador de severidade de flicker de curta duração.

\section{CONCLUSÕES}

O presente trabalho mostrou que existem deficiências intrínsecas na metodologia desenvolvida pela IEC (IEC, 2003) na quantificação dos níveis de flicker produzidos quando da presença de componentes de tensão com frequências interharmônicas sobrepostas à tensão fundamental. A primeira deficiência verificada decorre da propriedade natural do algoritmo $R M S$ de modulação do valor da tensão eficaz quando da presença de componentes inter-harmônicas de frequências elevadas. Nesse sentido, demonstrou-se que as maiores amplitudes de modulação do valor eficaz da tensão ocorrem para as frequências imediatamente adjacentes à frequência fundamental. Outra deficiência verificada está relacionada com a estrutura do flickermeter IEC, particularmente em função dos filtros digitais que compõem o bloco 3 do referido protocolo. Sobre esse aspecto, demonstrou-se que o filtro passa-baixa, tipo Butterworth de $6 \underline{a}$ ordem, impossibilita a correta quantificação dos indicadores de flicker quando da presença de tensões inter-harmônicas, com frequências superiores a 102 $H z$, sobrepostas à tensão fundamental.

Finalmente, o presente trabalho fez uma referência ao fato de que a flutuação do fluxo luminoso em lâmpadas de filamento está diretamente relacionada com a modulação do valor eficaz da tensão imposta. Porém, no caso das lâmpadas fluorescentes compactas, a flutuação do fluxo luminoso está mais fortemente relacionada com a modulação dos valores de pico da tensão aplicada. Nesse sentido, foram apresentados resultados de testes experimentais, segundo os quais os fluxos luminosos, produzidos por lâmpadas incandescentes e fluorescentes compactas, foram analisados quando da sobreposição de componentes inter-harmônicas ao sinal de tensão de alimentação das referidas lâmpadas. Os resultados obtidos demonstraram que lâmpadas fluorescentes compactas, contrariamente às lâmpadas incandescentes, podem apresentar cintilação luminosa quando da sobreposição de inter-harmônicas de frequências elevadas. 


\section{REFERÊNCIAS}

Gallo, D., Landi, C., Langella, R. and Testa, A. (2004). Iec flickermeter response to interharmonic pollution, Harmonics and Quality of Power, 2004. 11th International Conference on, pp. 489 - 494.

IEC (2003). Electromagnetic compatibility (emc) - part 4: Testing and measurement techniques - section 15: Flickermeter - functional and design specifications.

IEEE (2001). Interharmonic task force working group document draft 3, Technical report, IEEE.

URL: http://grouperieee.org/groups/harmonic/iharm/docs/ ih519c.pdf
Li, C., Xu, W. and Tayjasanant, T. (2003). Interharmonics: basic concepts and techniques for their detection and measurement, Electric Power Systems Research 66(1): 39 - 48. <ce:title>Power Quality</ce:title>.

URL: http://www.sciencedirect.com/science/article/pii/ S0378779603000701

Macedo Jr., J. R. and Simonetti, D. S. L. (2009). As interharmônicas e o fenômeno da cintilação luminosa, VIII Conferência Brasileira sobre Qualidade da Energia Elétrica-CBQEE 2009, Blumenau, SC. 